\title{
Supporting information: Phase field modelling of morphologies driven by tetragonal interfacial energy anisotropy
}

\author{
Arijit Roy ${ }^{*, \dagger} \ddagger$ and M.P. Gururajan ${ }^{\dagger}$ \\ $\dagger$ Department of Metallurgical Engineering and Materials Science, Indian Institute of \\ Technology Bombay, Powai, Mumbai 400076 INDIA. \\ $\ddagger$ Current address: School of Materials Science and Engineering, Kookmin University, Seoul \\ 02707, Republic of Korea \\ E-mail: arijitroy6@gmail.com
}


This document consists of two parts. In the first part, we describe the equations along with an example GNU Octave script to draw the 3D polar plots shown in the paper. In the second part, we describe the construction of inner envelope of the free energy polynomial sections to understand the equilibrium morphologies of the precipitates.

\section{GNU Octave script to draw the 3D polar plots}

An example GNU Octave script to draw the 3D polar plots as shown in the paper is attached in this supplementary document. A brief discussion on the equations used in this script file is presented. In order to draw the 3D surface plot, we define the direction cosine in terms of the polar $(u)$ and azimuthal $(v)$ angles in the spherical co-ordinate system. We choose polar

angle to vary from $-\frac{\pi}{2}$ to $+\frac{\pi}{2}$ and azimuthal angle is varying from $-\pi$ to $+\pi$. In that case the direction cosines along $\widehat{x}, \widehat{y}$, and $\widehat{z}$ can be written as,

$$
\begin{aligned}
& \widehat{c_{1}}=\cos (u) \sin (v) \\
& \widehat{c_{2}}=\cos (u) \cos (v) \\
& \widehat{c_{3}}=\sin (u),
\end{aligned}
$$

respectively. The tensorial polynomial of $\operatorname{rank} 2,4$, and 6 are defined in terms of $P 2, P 4$, and $P 6$ respectively. Finally, the complete polynomial (presented by $P$ in the script below) is written by scaling each of the above mentioned polynomial power $\frac{1}{\text { rank }}$ and summing them all together;

$$
P=P 2^{1 / 2}+P 4^{1 / 4}+P 6^{1 / 6}
$$

In order to draw the surface plot using surf command, we need to change the co-ordinate from spherical (in terms of $u, v$, and $R$ ) to Cartesian (in terms of $X, Y$, and $Z$ ) using sph2cart function. The detailed script with the complete printing set up is as follows:

clear all; 
clf ;

$\mathrm{nsize}=50$

$\% * * * * * * * * * * * * *$ Plot set up $* * * * * * * * * * *$

$\mathrm{h}=$ get $($ gcf, "currentaxes ");

set (h, 'Fontsize', 50 , " fontweight ", "normal ", " linewidth ",2)

set (gcf, 'PaperSize', [ [12.5 12.5$]$ );

set (gcf, 'PaperPositionMode', 'manual');

set (gcf, 'PaperPosition', , [ [ l $\left.\left.\begin{array}{llll}0.05 & 0 & 12.45 & 12.5\end{array}\right]\right)$;

colormap (jet)

$\mathrm{p} 1=1.0 ; \mathrm{p} 2=0.27 ;$

$\mathrm{m} 1=0 ; \mathrm{m} 2=0 ; \mathrm{m} 3=0 ; \mathrm{m} 4=0 ;$

$\mathrm{n} 1=100 ; \mathrm{n} 2=27 ; \mathrm{n} 3=800 ;$

$\mathrm{n} 4=1000 ; \mathrm{n} 5=600 ; \mathrm{n} 6=-3900 ;$

$\% \% 3 \mathrm{D}$ polar plot

pol $=\operatorname{linspace}(-$ pi $/ 2$, pi $/ 2$, nsize $)$;

azimuthal $=$ linspace $(-$ pi, pi, nsize $)$;

$[\mathrm{u}, \mathrm{v}]=\operatorname{meshgrid}($ pol, azimuthal $)$;

$\mathrm{c} 1=\cos (\mathrm{u}) \cdot * \sin (\mathrm{v})$;

$\mathrm{c} 2=\cos (\mathrm{u}) \cdot * \cos (\mathrm{v})$;

$\mathrm{c} 3=\sin (\mathrm{u})$;

$\mathrm{P} 2=\mathrm{p} 1 *(\mathrm{c} 1 * \mathrm{c} 1+\mathrm{c} 2 * \mathrm{c} 2)+\mathrm{p} 2 *(\mathrm{c} 3 * \mathrm{c} 3)$

$\mathrm{P} 4=\mathrm{m} 1 *\left(\mathrm{c} 1 \bullet^{\wedge} 4+\mathrm{c} 2 \cdot{ }^{\wedge} 4\right)+\mathrm{m} 2 * \mathrm{c} 3 . \wedge 4+\ldots$

$2 * \mathrm{~m} 3 *\left(\left(\mathrm{c} 1 .{ }^{\wedge} 2\right) \cdot *\left(\mathrm{c} 2 .{ }^{\wedge} 2\right)\right)+2 * \mathrm{~m} 4 *(\mathrm{c} 3 . \wedge 2) \cdot *\left(\mathrm{c} 1 .{ }^{\wedge} 2+\mathrm{c} 2 . \wedge 2\right)$;

$\mathrm{P} 6=\mathrm{n} 1 *\left(\mathrm{c} 1 .{ }^{\wedge} 6+\mathrm{c} 2 \cdot{ }^{\wedge} 6\right)+\mathrm{n} 2 * \mathrm{c} 3 .{ }^{\wedge} 6+\ldots$

$\mathrm{n} 3 *(\mathrm{c} 1 . \wedge 2) \cdot *\left(\mathrm{c} 2 \cdot{ }^{\wedge} 2\right) \cdot *(\mathrm{c} 1 . \wedge 2+\mathrm{c} 2 . \wedge 2)+\ldots$

$\mathrm{n} 4 *(\mathrm{c} 3 . \wedge 2) \cdot *\left(\mathrm{c} 1 . \wedge 4+\mathrm{c} 2 \cdot{ }^{\wedge} 4\right)+\ldots$

$\mathrm{n} 5 *\left(\mathrm{c} 3 .{ }^{\wedge} 4\right) \cdot *\left(\mathrm{c} 1 \cdot^{\wedge} 2+\mathrm{c} 2 \cdot{ }^{\wedge} 2\right)+\ldots$ 


$$
\begin{gathered}
\mathrm{n} 6 *((\mathrm{c} 1 . \wedge 2) \cdot *(\mathrm{c} 2 \cdot \wedge 2) \cdot *(\mathrm{c} 3 . \wedge 2)) \\
\mathrm{P}=(\mathrm{P} 2 . \wedge(1.0 / 2))+(\mathrm{P} 4 . \wedge(1.0 / 4))+(\mathrm{P} 6 . \wedge(1.0 / 6))
\end{gathered}
$$

hold on

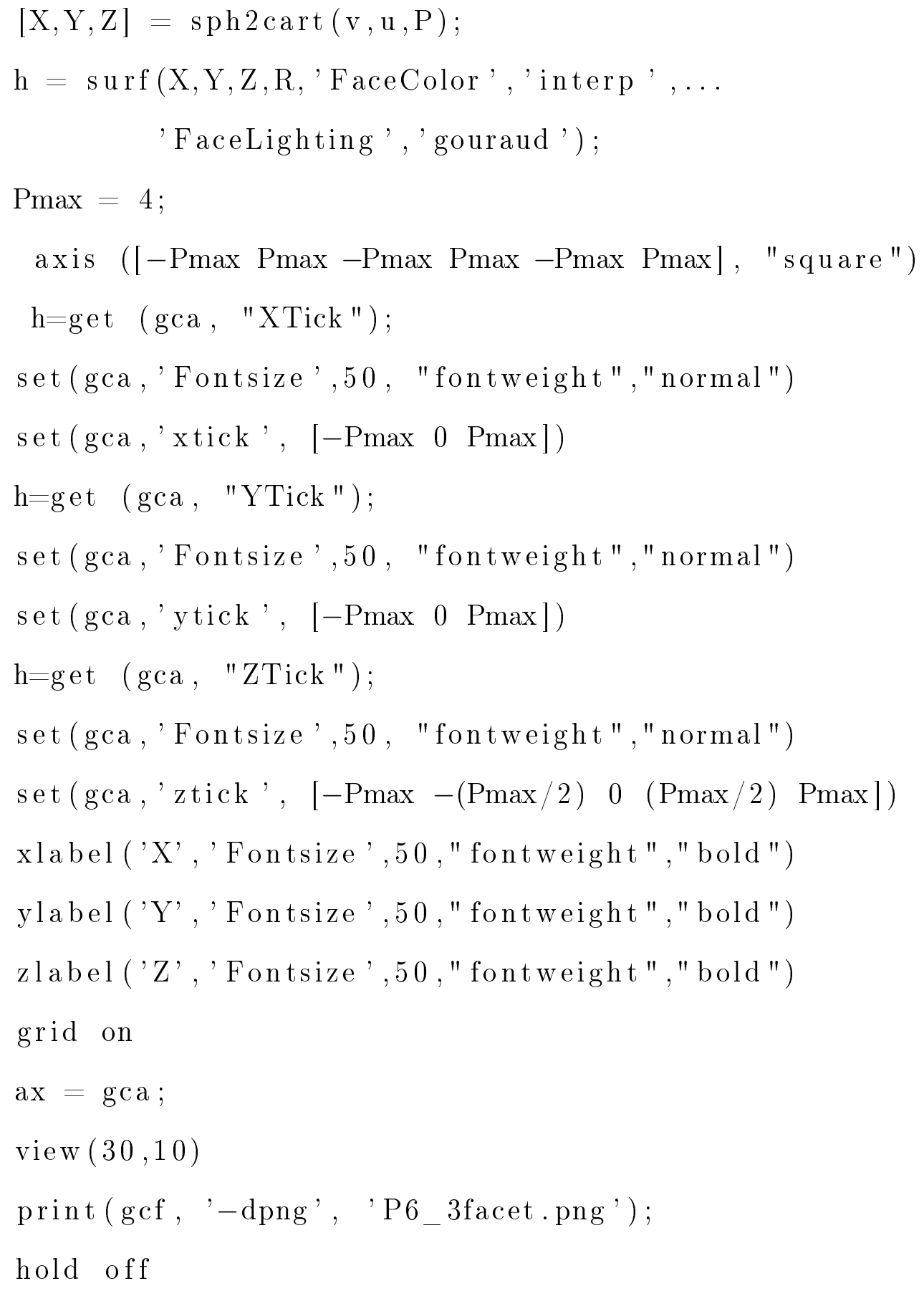




\section{How to draw the inner envelope, mimicking the possible equilibrium shape of the precipitate?}

We construct the inner envelope on the sections of anisotropic polynomial using the following relations:

$$
\begin{aligned}
& x=P(\theta) \cos (\theta)-\frac{d P(\theta)}{d \theta} \sin (\theta) \\
& y=P(\theta) \sin (\theta)+\frac{d P(\theta)}{d \theta} \cos (\theta) .
\end{aligned}
$$

Here $P(\theta)$ is the orientation dependent polynomial (as described by Eq. 1 in the previous section) and $\theta$ is the varying orientation angle on a specific plane. Note that a section of crystallographic plane can be defined using either of the angles - polar or azimuthal - by fixing either of them to be a constant. For example, we need to fix the polar angle to $u=0$ and vary azimuthal angle from $-\pi$ to $+\pi$ to obtain a circular cross-section on the (100) plane. In order to draw the inner envelope on such polynomial cross section, the varying angle $\theta$ in the above equations will follow the variation in azimuthal angle.

\section{Tabulation of the polynomial coefficients used to draw the 3D morphologies}

We tabulate the polynomial coefficients used to draw the 3D morphologies in Fig. 7, 10, and 12 in Table. 1. 
Table 1: Table for polynomial coefficients used to generate the 3D morphologies shown in Fig. 7, 10, and 12.

\begin{tabular}{cccccc}
\hline \hline Coefficients & Fig. 7(a) & Fig. 7(b) & Fig. 7(c) & Fig. 10 & Fig. 12 \\
\hline \hline$p_{1}$ & 1.0 & 2.0 & 1.0 & 1.0 & 1.0 \\
$p_{2}$ & 1.0 & 0.1 & 2.0 & 2.4 & 0.27 \\
\hline$m_{1}$ & 200.0 & 200.0 & 300.0 & 0 & 0 \\
$m_{2}$ & 1000.0 & 10.0 & 700.0 & 0 & 0 \\
$m_{3}$ & -30.0 & -90.0 & 1050.0 & 0 & 0 \\
$m_{4}$ & 291.0 & 100.0 & 100.0 & 0 & 0 \\
\hline$n_{1}$ & 0 & 0 & 0 & 100.0 & 100.0 \\
$n_{2}$ & 0 & 0 & 0 & 1200.0 & 27.0 \\
$n_{3}$ & 0 & 0 & 0 & 5300.1 & 800.0 \\
$n_{4}$ & 0 & 0 & 0 & 6000.0 & 1000.0 \\
$n_{5}$ & 0 & 0 & 0 & 1000.0 & 600.0 \\
$n_{6}$ & 0 & 0 & 0 & -3000.0 & -3900.0 \\
\hline \hline
\end{tabular}

\section{Visualization of three different types of facets as discussed}

\section{in Fig. 12}

Three different family of facets, (100), (001), and (111) as discussed in Fig. 12 of the main manuscript are shown in Fig. 1.
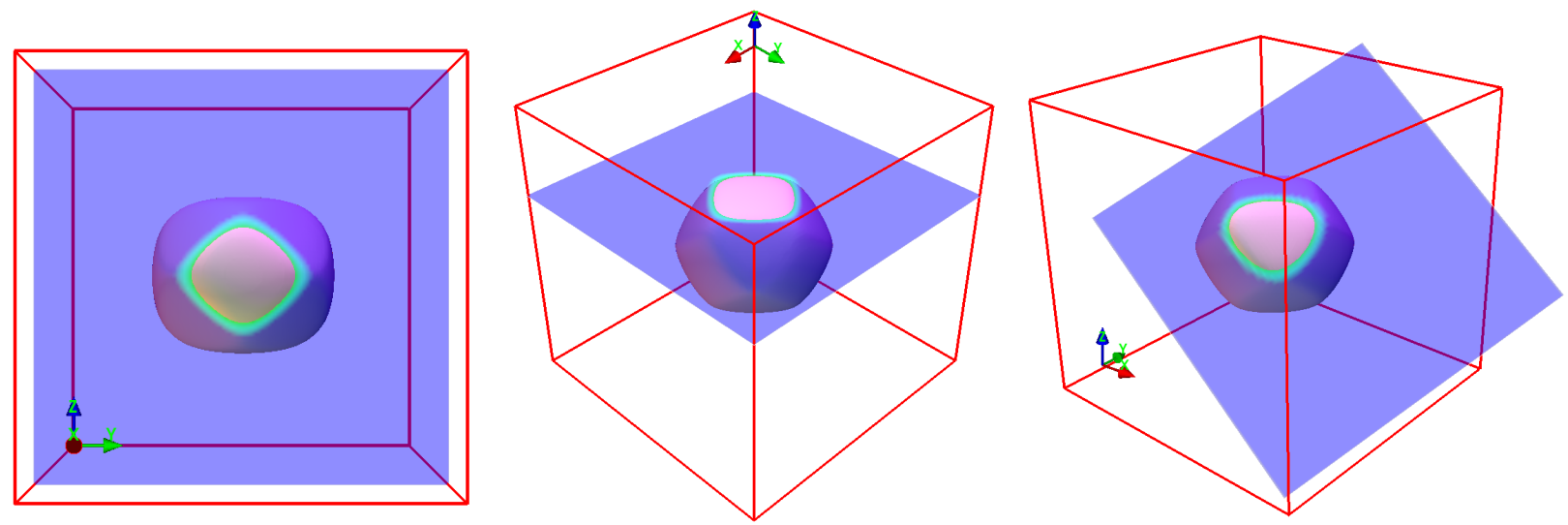

Figure 1: Three different cut planes of $\{100\}$ (left figure), $\{001\}$ (middle figure), and $\{111\}$ (right figure) are drawn for the easy understanding of the facets as appear in Fig. 12 of the main manuscript. 